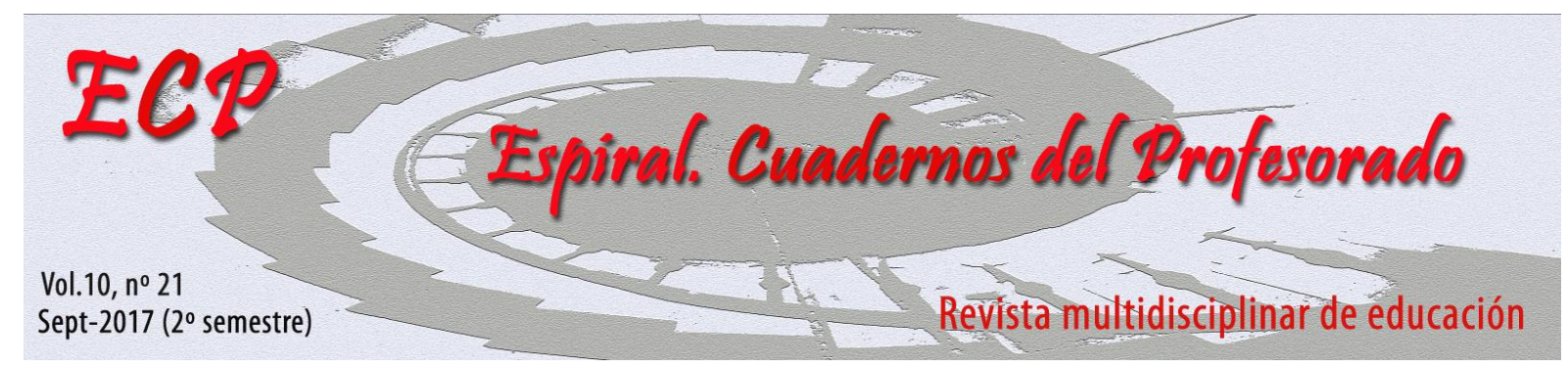

\title{
La poesía de Joan Margarit para nivel avanzado de EEOOII en E/LE: una propuesta didáctica
}

\section{Poetry for advanced level of Spanish as a foreign language in the Official Schools of Languages: a didactic proposal}

\section{Raquel Lanseros Sánchez ${ }^{1}$ y María Remedios Sánchez García ${ }^{2}$}

1. Departamento de Didáctica de la Lengua y la Literatura, Universidad de León, España

2. Departamento de Didáctica de la Lengua y la Literatura, Universidad de Granada, España

\begin{abstract}
RESUMEN: El presente trabajo es una propuesta didáctica de aplicación de la poesía al aula de español como lengua extranjera, en el nivel avanzado de las Escuelas Oficiales de Idiomas. Se pretende que el alumnado desarrolle su competencia lectora y escritora, así como sus conocimientos sobre el fenómeno poético y el contexto cultural de la lengua española. Para ello, se ha seleccionado un poema de Joan Margarit, cuya temática aborda la historia reciente de España, a fin de crear un espacio de reflexión sobre los acontecimientos que han marcado el presente de nuestro país. A partir del poema, se propone una serie de actividades que potencian el desarrollo de las cuatro destrezas comunicativas, así como la interpretación del lenguaje poético y su multiplicidad de significación. A través de la enseñanza lingüística, se persigue lograr un mayor conocimiento de la poesía contemporánea escrita en español y un estímulo al fomento de la lectura.
\end{abstract}

Palabras clave: Poesía, Joan Margarit, nivel avanzado de las EEOOII, educación literaria, español como lengua extranjera.

\begin{abstract}
The present work is a didactic proposal of application of poetry to the classroom of Spanish as a foreign language, in the advanced level of the Official Schools of Languages. It is intended that the students develop their reading and writing skills, as well as their knowledge of the poetic phenomenon and the cultural context of the Spanish language. For this purpose, a poem by Joan Margarit has been selected, whose subject addresses the recent history of Spain, in order to create a space for reflection on the events that have marked the present of our country. From the poem, a series of activities are proposed, meant to enhance the development of the four communicative skills, as well as the interpretation of poetic language and its multiplicity of meaning. Through linguistic teaching, the aim is to achieve a greater knowledge of contemporary poetry written in Spanish and a stimulus to the promotion of reading.
\end{abstract}

Key words: Poetry, Joan Margarit, advanced level of Official Schools of Languages, literary education, Spanish as a foreign language.

Lanseros Sánchez, R. y Sánchez García, M. R. (2017). La poesía de Joan Margarit para nivel avanzado de EEOOII en E/LE: una propuesta didáctica. Espiral. Cuadernos del Profesorado, $10(21), 90-99$. 


\section{Introducción}

La poesía es una de las principales herramientas didácticas con las que cuenta el profesor en el aula de lengua extranjera. Por su creatividad, su uso estético del lenguaje y su multiplicidad de sentidos, la poesía supone una fuente imprescindible de aprendizaje, tanto en el ámbito lingüístico como en el cultural. Como afirma José Luis Toscano "debemos reivindicar el gran potencial de la poesía en una enseñanza de ELE comunicativa y personalizada, precisamente por su eficacia expresiva y por fomentar la creatividad del individuo" (2013, p. 115).

El conocimiento de la cultura que rodea a la lengua extranjera que desea adquirirse, y más concretamente de su literatura, ayuda a desarrollar una valoración más positiva de ella, además de hacer al estudiante partícipe de conocimientos que favorecen su proceso de aprendizaje. Este refuerzo de la ligazón afectiva del alumno hacia la lengua que estudia se produce de una forma decidida mediante el uso y análisis de la poesía en el aula, puesto que ésta potencia inferencias estéticas de índole universal que facilitan el funcionamiento de la memoria y la individualización del vocabulario y las estructuras lingüísticas.

Además, la exposición a los textos poéticos genera una respuesta emocional muy favorecedora del proceso de aprendizaje, alejándolo de las propuestas gramaticalistas conservadoras, más frías y rutinarias. Es innegable además, como apunta Albadalejo "la necesidad de una revalorización de su potencial como fuente de inspiración para la creación de actividades comunicativas, a la vez que integradoras de las cuatro destrezas lingüísticas" (2007, p. 2). Se busca darles un mayor protagonismo, convertirlos en epicentro de las propuestas didácticas, a fin de desarrollar las destrezas del alumnado, evitando lo que ya apuntaba Acquaroni:

[...] situar el poema al final de la unidad está revelándonos, en muchos casos, las dificultades que dicho material plantea al profesor para incorporarlo realmente en el desarrollo de la clase: el poema queda así relegado a una posición de cierre marginal, de ejercicio voluntario que generalmente y por motivos de tiempo para el cumplimiento de la programación no se llega a realizar nunca en clase" (1997, p. 19).

Es muy importante que la presentación se realice utilizando una metodología activa y dinámica tal y como sugieren Acquaroni (2000) y Cassany (2006), alejada del modelo lingüístico y normativo para los estudiantes. En este sentido, es de fundamental importancia el trabajo investigador López Valero, cuyos talleres desarrollan la escritura creativa, la enseñanza de la lengua y la literatura y la expresión oral y escrita. Se trata de un indispensable vehículo de conocimiento cultural y un motivador instrumento de trabajo, en virtud del atractivo que posee para el estudiante.

Para poder sacar partido de estos objetivos, primero hemos de vincular el uso de la poesía en el aula de español como lengua extranjera con los intereses del alumnado. Desde una perspectiva de desarrollo de la competencia intercultural de la que ya hablan Alonso y Fernández-Agüero (2013). Creemos que la presentación de formas más elaboradas y artísticas de la lengua española, como son los poemas, servirán de instrumento de conocimiento amplio de la cultura, así como de reflexión lingüística. Algunos de estos objetivos que alcanzaremos con la introducción de la poesía en el aula de español como lengua extranjera son los siguientes:

- Fomentar la capacidad de distinción entre los significados literales y simbólicos o metafóricos.

- Proporcionar una visión más profunda de la compleja realidad que supone toda lengua.

- Motivar al alumnado mediante la exposición de textos de gran contenido artístico.

- Ampliar el corpus de vocabulario utilizado, preferentemente en los cursos avanzados.

\footnotetext{
${ }^{1}$ El taller de lengua y literatura: (cien propuestas experimentales) (1993), de Amando López Valero y Pedro Guerrero Ruiz fue una obra pionera a la hora de retomar el modelo metodológico de Giani Rodari. López Valero, junto a Encabo Fernández, ha propuesto también un modelo heurístico de comunicación para optimizar el proceso de enseñanza-aprendizaje en el aula, en Heurística de la comunicación: el aula feliz (2001).
} 
- Profundizar en el conocimiento de la cultura propia de los países de habla hispana.

- Despertar el interés por la poesía en español más allá de nuestras fronteras.

\section{Joan Margarit}

Joan Margarit es un poeta excepcional en el sentido literal del término, ya que sus circunstancias vitales lo conforman como un creador único, cuya trayectoria literaria difícilmente puede ser comparada con ningún otro de sus coetáneos. La calidad y originalidad de la obra de Joan Margarit, además de aportar a la poesía española contemporánea la óptica de un poeta que escribe en catalán y en español -con toda la riqueza intrínseca que ello conlleva-, supone asimismo la experiencia poética de un autor cuya profesión es la arquitectura, hecho que contribuye asimismo a expandir el ángulo de visión de la poesía.

La trayectoria poética de Joan Margarit posee una singularidad tal en desarrollo, temática, afinidades y estructura, que no caben adscripciones generacionales ni compartimentos poéticos para explicar su trayecto creativo. El poeta refleja la memoria en dos lenguas en las cuales él va construyéndose una educación literaria que viene a marcar toda su cosmogonía. Dueño de una pluma limpia, de trazo transparente pero con hondas reverberaciones, su estilo aparentemente sencillo ha cautivado a generaciones de lectores, trasladando un hondo sentido del paso del tiempo y un hedonismo dulcificado por un agudo sentimiento de compasión y ternura hacia los otros.

Algunas de sus obras más conocidas y celebradas son Joana (2002), Cálculo de estructuras (2005), Casa de misericordia (2007) - por el cual fue galardonado con el Premio Nacional de Poesía-, Misteriosamente feliz (2009) o Amar es dónde (2015). Su obra poética ha sido reunida íntegramente en el volumen Todos los poemas (1975- 2012) (2015), con prólogo de José Carlos Mainer.

\section{¿Por qué Joan Margarit?}

Hemos seleccionado un poema de Joan Margarit para efectuar nuestra propuesta didáctica. Se trata de familiarizar al alumnado con el lenguaje poético y, más allá, con el universo literario de Joan Margarit, rico en alusiones histórico-biográficas y en reflexiones vitales.

Para la selección de este poema, hemos tenido en cuenta el especial significado simbólico dentro de su trayectoria vital y poética. "Casa de misericordia" le da título al libro al que pertenece, por el que Joan Margarit recibió el Premio Nacional de Poesía de España en el año 2008. El poeta se refiere a la Guerra Civil española y los durísimos tiempos de posguerra que le siguieron. Sus recuerdos de aquella terrible época están constantemente presentes en su poesía. El texto estimulará el conocimiento de la historia reciente de España por parte de los estudiantes de español como lengua extranjera, coadyuvando a su enriquecimiento cultural, además de lingüístico. La empatía es una de las señas literarias de identidad de Joan Margarit, desde la cual el poeta crea ese territorio hospitalario y acogedor que son sus poemas. La honestidad y la verdad son dos características fundamentales para Margarit a la hora de enfrentarse a la construcción de su obra creativa y de definir su visión del mundo. Él mismo afirma:

Siempre he tenido conciencia de que, para mí, la poesía se extendía por toda la vida. La prisa, pues, no ha formado parte de mi relación con el poema. El juicio final lo hará el tiempo y, al contrario de los juicios finales de las religiones, yo no sabré el resultado. A mí me corresponde sólo -y no es poco- el día a día con los poemas sin más justificación, placer o compensación que buscarlos, componerlos y escribirlos. Ninguno de nosotros contamos mucho, incluso los que parecen contar mucho, pero nos puede salvar lo mismo que, curiosamente, también puede salvar el poema: su honesta intensidad ${ }^{2}$.

\footnotetext{
${ }^{2}$ Extracto del prólogo a la primera edición de Tots els poemes 1975-2012, Ediciones 62, Colección Labutxaca, 2013.
} 


\section{Propuesta didáctica a partir de "CASA DE MISERICORDIA", un poema de CASA DE MISERICORDIA, de Joan Margarit}

\section{Nivel de aplicación y temporalización}

La poesía aúna, dentro de los géneros literarios, todas las funciones comunicativas que explicitara Roman Jakobson. Su inclusión en el aula de español para extranjeros de las EEOOII es imprescindible para dotar a los alumnos de una formación linguística que incluya contenidos socioculturales y desarrollo de la educación emocional en la lengua de aprendizaje. El propio Marco común europeo de referencia para las lenguas (MCER) lo explicita:

Los estudios literarios cumplen muchos más fines educativos, intelectuales, morales, emocionales, lingüísticos y culturales que los puramente estéticos. (200, p. 60).

Conscientes de que su comprensión requiere unos conocimientos previos amplios de la lengua española, hemos situado nuestra propuesta didáctica en el Nivel Avanzado de la Enseñanza de Idiomas de Régimen Especial, del idioma español para extranjeros. Se trata del nivel más alto, dentro de la enseñanza de idiomas de régimen especial, y es, por tanto, el nivel más adecuado para la explotación didáctica del poema propuesto. La presente propuesta didáctica puede ser implementada en el primer o segundo curso del nivel avanzado, dependiendo del criterio personal del profesor y el grado global de conocimiento de cada grupo determinado.

Según el Real Decreto 1629/2006, de 29 de diciembre, por el que se fijan los aspectos básicos del currículo de las enseñanzas de idiomas de régimen especial reguladas por la Ley Orgánica 2/2006, de 3 de mayo, de Educación; la definición del nivel avanzado es la siguiente:

El Nivel avanzado presentará las características del nivel de competencia B2, según este nivel se define en el Marco Común Europeo de Referencia para las Lenguas (MCER).

El nivel avanzado tiene como finalidad capacitar a los alumnos para utilizar el idioma con soltura y eficacia en situaciones habituales y más específicas que requieran comprender, producir y tratar textos orales y escritos conceptual y lingüísticamente complejos, en una variedad de lengua estándar, con un repertorio léxico amplio aunque no muy idiomático, y que versen sobre temas generales, actuales o propios del campo de especialización del hablante ${ }^{3}$.

Esta propuesta didáctica se desarrollará en un promedio de dos o tres sesiones, de modo que abarquen entre una hora y cuarenta minutos y dos horas y media de trabajo en común -si tenemos en cuenta que cada sesión académica tiene una duración de cincuenta minutos- más el trabajo individual realizado por cada alumno fuera del horario lectivo.

\section{Aclaraciones metodológicas}

La metodología empleada tendrá un enfoque comunicativo y velará por la potenciación y el desarrollo de las cuatro destrezas comunicativas: comprensión oral, comprensión escrita, expresión e interactuación oral y expresión e interactuación escrita. Se tratará en todo momento de estimular una comprensión profunda del texto a través de la lectura y su función social y a la par lúdica, en la línea de lo expuesto para otros niveles por Cerrillo Torremocha (2015). Como afirma Sáiz-López:

Leyendo se adquieren conocimientos de las áreas convencionales del saber, así como de aspectos, fenómenos y facetas de otras sociedades, entre otras cosas. La obra literaria, una elaboración creativa en unos parámetros socioculturales concretos, (nos) propone un conjunto de pensamientos, sensaciones y acontecimientos sujetos a una mirada particular y, más o menos, subjetiva que tendemos a considerar como un ejercicio cultural en el doble sentido del término, como actividad y como representación. (2015, p. 131).

\footnotetext{
${ }^{3}$ Otro interesante trabajo reciente sobre el uso de la poesía en el aula de lengua es el artículo de Bianca Estela Sánchez Pacheco, "Nuevas tendencias poéticas en el aula: poesía ante la incertidumbre como herramienta didáctica" (2016), publicado en el número 14 de la revista Álabe.
} 

elementos:

La aproximación a la didáctica de la poesía ha de ser interactiva e incluir los siguientes

- Enfoque comunicativo

- Clases prácticas, ágiles e innovadoras

- Interpretación conjunta de los textos

- Interacción continua profesor-alumno

- Aprendizaje cooperativo

- Trabajo escrito e iniciación a la escritura creativa

- Debate abierto sobre los múltiples sentidos y significaciones de la poesía

- Análisis de los elementos culturales presentes

- Uso de las TIC (plataformas, páginas web, blogs, webquests, etc.) para recabar y ampliar información

En cuanto a la aplicación de la poesía al aula, es importante que el profesor no actúe tan sólo como transmisor de información, sino que se convierta en investigador de la propia acción educativa que está poniendo en práctica. De este modo, pasará a ser mediador entre su propia teoría y la praxis educativa implementada según aclara también Cerrillo Torremocha (2004, p. 247-260).

\section{Planteamiento inicial}

El poema "Casa de misericordia" le da título al libro al que pertenece, por el que el poeta Joan Margarit recibió el Premio Nacional de Poesía de España en el año 2008.

Es importante realizar como paso inicial una contextualización histórico-literaria del poeta cuyo poema seleccionado vamos a ir trabajando. En este sentido, Quiles Cabrera y Caire (2013) afirman:

La idea de integración que hemos asociado a la competencia cultural en los contextos de inmigración aludidos ha de ampliarse al proceso de inmersión del estudiante de nivel avanzado que pretende ser especialista en la didáctica del español. En este sentido, al conocimiento de las convenciones sociales que rigen determinados usos lingüísticos habremos de sumar un contacto completo con los procesos históricos, con los cambios sociales y las producciones artísticas propias de la comunidad de la lengua meta.

Por ello, días antes de comenzar la actividad, deberemos proporcionar a los alumnos algunos enlaces y páginas web donde buscar información sobre el poeta y su obra.

Para el día del comienzo de la actividad didáctica con el poema "Casa de misericordia", les habremos pedido que traigan redactada una pequeña biografía del autor, en la que incluyan lo más representativo de su vida y su obra.

Comenzaremos la actividad leyendo en grupo unas cuantas biografías del poeta escritas por los propios alumnos. El profesor aclarará aquellos detalles o aspectos tanto de su vida como de su obra poética que hayan podido pasar desapercibidos o no hayan sido comprendidos correctamente.

Una vez familiarizados con la figura del autor, procedemos a la lectura individual y grupal de su poema "Casa de misericordia".

\section{CASA DE MISERICORDIA}

El padre fusilado.

$\mathrm{O}$, como dice el juez, ejecutado.

La madre, ahora, la miseria, el hambre,

la instancia que le escribe alguien a máquina:

Saludo al Vencedor, Segundo Año Triunfal,

Solicito a Vuecencia poder dejar mis hijos

en esta Casa de Misericordia. 
El frío del mañana está en la instancia.

Hospicios y orfanatos fueron duros, pero más dura era la intemperie.

La verdadera caridad da miedo.

Igual que la poesía: un buen poema,

por más bello que sea, será cruel.

No hay nada más. La poesía es hoy

la última casa de misericordia.

\section{Actividades}

Para comenzar, los alumnos leerán en voz baja el poema "Casa de misericordia", fijándose especialmente en las palabras, las construcciones gramaticales, sus imágenes y las sensaciones que los versos les provocan. Seguidamente, en dos grandes grupos, intentarán repetir la primera y la segunda estrofa del poema, cuidando todos los detalles que tienen que ver con la pronunciación y la entonación. Después, cada grupo designará un portavoz que se encargará de declamar en voz alta para la clase la estrofa correspondiente de las dos trabajadas.

A continuación, los alumnos buscarán en el diccionario las palabras que no comprendan. Una vez cerrados los diccionarios, y buscadas todas las palabras desconocidas, tratarán de escribir su propia definición de los siguientes vocablos: Fusilado, instancia, triunfal, solicitar, vuecencia, hospicio, orfanato, intemperie. Asimismo, intentarán buscar un sinónimo en español para cada una de las palabras anteriores.

Se invitará a los alumnos a tratar de adivinar qué es una casa de misericordia. Buscarán información y harán una pequeña redacción de cuarenta palabras explicando qué son estos establecimientos, cuándo comenzaron a fundarse, cuál era su misión y en cuántos países han existido.

Seguidamente, busca la palabra "misericordia" en el diccionario de la lengua española, de la Real Academia Española de la lengua. Encontrarán cinco acepciones diferentes y habrán de decidir cuál de ellas es la más apropiada para el contexto del poema. Para ello, leerán en clase previamente en voz alta cada una de las cinco acepciones de la palabra "misericordia". Debatirán conjuntamente sobre cuál es la acepción más cercana al contexto del poema, razonando su respuesta y argumentando las razones para haber elegido una u otra.

La primera estrofa del poema está íntegramente escrita en tiempo presente. Tan sólo el segundo y el tercer poema de la segunda estrofa cambian bruscamente a tiempo pasado. Se pedirá a los alumnos que traten de explicar por qué sucede esto, así como qué creen que el poeta desea transmitir con esta combinación de tiempos verbales.

A continuación, releerán el verso: "Solicito a Vuecencia poder dejar mis hijos". El término "vuecencia" es una fórmula de tratamiento arcaica que apenas se usa hoy en día fuera del ambiente castrense. Se preguntará a los alumnos sobre el tipo de relación jerárquica entre personas que indica este uso. También se debatirá sobre por qué creen que la madre a la que se refiere el poema se ve obligada a utilizarlo.

Se les pedirá, acto seguido, que lean con atención el siguiente verso: "Saludo al Vencedor, Segundo Año Triunfal"

En su opinión, ¿qué puede querer decir en este contexto la palabra "vencedor"? Se hablará en clase sobre si los alumnos conocen la guerra a la que el poeta se refiere y sobre qué puede significar la expresión "segundo año triunfal". Teniendo en cuenta el momento en que esta expresión se utilizaba, ¿en qué año creen los alumnos que tienen lugar los sucesos que relata el poema?

Joan Margarit afirma en el poema que "La poesía es hoy / la última casa de misericordia". Los alumnos debatirán a qué creen que el poeta se refiere mediante este símil. A su vez, argumentarán si, en su opinión, la poesía puede ser un lugar de acogida.

Se encomendará a los alumnos explicar en una redacción breve de unas cincuenta palabras el significado de la poesía para ellos, su lugar en su vida y las implicaciones emocionales o intelectuales que tiene para ellos 
Para finalizar, se les propondrá una actividad optativa. Si les ha gustado "Casa de misericordia", pueden buscar información sobre Casa de misericordia, el libro al que pertenece el poema de Joan Margarit, para compartirla en clase con sus compañeros. Pueden contarles en qué año se publicó, qué ha dicho la crítica de él, de cuántas partes se compone, etc. Pueden también seleccionar otro poema del libro que les guste y traerlo a clase para leerlo en voz alta.

\section{Criterios de evaluación}

El tratamiento especial que merece la poesía en el aula requiere que su aplicación didáctica no sea evaluable del mismo modo que el resto de contenidos gramaticales o léxicos. De ser así, perdería gran parte del atractivo que posee para el alumno. Hay que recordar que la meta final no es aprender de memoria la biografía de un autor ni una lista de vocabulario, sino interpretar todos los posibles sentidos que tiene el lenguaje utilizado artísticamente y abrir la mente al mundo cultural que subyace bajo las palabras del idioma en curso de aprendizaje.

Los criterios de evaluación del nivel avanzado de español como lengua extranjera incluyen las competencias propias del nivel para cada una de las cuatro destrezas: comprensión oral, comprensión escrita, expresión e interacción oral y expresión e interacción escrita.

Sin embargo, debemos tener siempre presente que la inclusión de la poesía en el aula no es una propuesta didáctica gratuita, sino muy enriquecedora. Y precisamente a causa de su especial carácter de delectación estética, consideramos que sería contraproducente proceder con el mismo esquema evaluativo aplicable al resto de unidades didácticas del curso.

De este modo, los criterios de evaluación particulares para esta propuesta didáctica serían independientes y estarían únicamente basados en el poema seleccionado y las actividades realizadas. Como ejemplo, los siguientes ítems pueden ser utilizados por el profesor para medir el rendimiento y la implicación de los alumnos en la propuesta didáctica de aplicación de la poesía al aula, puntuando cada uno de ellos como muy logrado, satisfactorio o no logrado.

1. ¿El estudiante muestra interés hacia la poesía española a través de Joan Margarit?

2. ¿Reconoce las características y los rasgos identificativos de la poesía de Joan Margarit?

3. ¿Mejora su comprensión lectora y su apreciación literaria con el desarrollo de la propuesta?

4. ¿Se observa un aprendizaje progresivo de las distintas habilidades comunicativas?

5. ¿Es capaz de llegar a una comprensión profunda del poema elegido?

6. ¿Logra entender el significado de las palabras por su contexto?

7. ¿Mediante la lectura comprende el texto planteado en clase y es capaz de ver la intertextualidad que de él se desprende?

8. ¿Participa activamente en los debates suscitados con la aplicación didáctica del poema?

9. ¿Es capaz de expresarse con propiedad utilizando todo lo aprendido?

10. ¿Se observa una actitud positiva hacia la potenciación de la creatividad en el aula?

11. ¿Es capaz de valorar los contenidos culturales y las propuestas estéticas contenidas en la poesía de Joan Margarit?

El presente cuestionario de evaluación es para uso interno del profesor, que podrá ampliarlo o modificarlo según las necesidades específicas de su grupo-clase. Es aconsejable rellenarlo a la finalización de la propuesta didáctica, tras el desarrollo del poema, para de este modo ser capaz de analizar mejor el rendimiento de cada uno de los alumnos.

Por tanto, se trata de una acción didáctica de carácter específico, en la cual no todos los contenidos han de ser evaluables, sino más bien ejercicios instrumentalizados, un incentivo extra para que el estudiante se sumerja en la cultura de la lengua objeto de aprendizaje, así como en su musicalidad y su potencialidad estética. Este tratamiento más distendido potenciará sin duda el 
atractivo que ejerce la poesía en el marco de enseñanza-aprendizaje del español como lengua extranjera.

\section{Procedimientos e instrumentos de evaluación}

Utilizaremos una serie de instrumentos de evaluación y de registro que especificamos a continuación, no sin antes mencionar algo que para nosotros resulta fundamental, y es la función mediadora del docente estructurando unos parámetros específicos centrados en el aprendizaje formativo aplicados a los objetivos que trabajamos en cada uno de los bloques de conocimiento. A partir de ahí, las herramientas fundamentales son:

Detección de conocimientos previos sobre el tema que vamos a tratar en el aula. Esta acción nos permite conocer el alcance del conocimiento anterior del alumnado, anticiparnos al grado de interés que suscita y realizar predicciones sobre la temporalización y los resultados.

El Diario de clase supondrá un registro de las diferentes actividades realizadas, las tareas desarrolladas y el grado de aprovechamiento y participación del alumnado.

Cuaderno de notas que refleja los resultados de los ejercicios y de las tareas realizados por los alumnos. Es un instrumento del proceso de evaluación formativa que facilita la posterior implementación del procedimiento de evaluación sumativa.

Actividades de comprensión lectora, escritura creativa y expresión oral susceptibles de ser evaluadas por el profesor, así como el esfuerzo y grado de aprovechamiento personal.

Encuestas de autoevaluación y de coevaluación, que nos ayudarán a detectar el grado de seguimiento de la propuesta didáctica por parte del alumnado.

Asimismo, es muy importante disponer de un cuestionario de evaluación propio, que deberá ser respondido una vez que la propuesta haya sido implementada en el aula, con vistas a asegurarnos una mejora de los aspectos cuyo proceso haya resultado menos satisfactorio, así como un ahondamiento en aquellos que más provecho hayan permitido extraer a los alumnos de la aplicación en el aula de la propuesta didáctica.

Algunas de las preguntas del cuestionario podrían ser:

- ¿Cuál de las actividades realizadas en esta propuesta didáctica ha funcionado mejor en el aula?

- ¿Qué parte del poema ha gustado más a los alumnos?

- ¿Son capaces los estudiantes de identificar las características personales de la poesía de Joan Margarit?

- ¿Ayuda la poesía a los estudiantes a mejorar su propia comprensión y reflexión sobre la existencia?

- ¿Se han asimilado los conceptos de cohesión, coherencia y adecuación tratados?

- ¿Han logrado los alumnos organizarse y trabajar de manera individual y en grupo?

- ¿Los debates realizados han contribuido a acercar posturas y a fomentar la tolerancia?

- ¿Las actividades de expresión oral ayudan a la evolución de los aspectos fónicos, gramaticales y léxicos de los estudiantes?

- ¿Han participado activamente los estudiantes en el desarrollo de la propuesta didáctica?

- ¿Hemos detectado alguna carencia significativa de la propuesta didáctica?

\section{Conclusiones}

Consideramos la poesía como una herramienta pedagógica indispensable en el aula de español como lengua extranjera. Su presencia en la programación didáctica del nivel avanzado de las EEOOII sería muy beneficiosa para el proceso de aprendizaje global del alumnado. Por un lado, se trata de un contacto lingüístico pluridimensional y altamente motivador. Por otro, posee una enorme 
potencialidad intrínseca para desarrollar la competencia comunicativa de los estudiantes, tanto en su vertiente lingüística como pragmática.

Para el presente trabajo de aplicación didáctica de la poesía de Joan Margarit al nivel avanzado del aula de español como lengua extranjera, hemos elegido un poema, con el cual hemos construido una propuesta didáctica no evaluable en términos convencionales, que constaría de tres a cuatro horas académicas, dependiendo del ritmo de aprendizaje de los estudiantes.

El enfoque comunicativo, predominante en la actualidad en las metodologías de enseñanza de lenguas extranjeras, subraya la importancia del uso de la poesía en el aula, como parte efectiva de la necesaria realia ${ }^{4}$ de la que hay que dotar al alumnado. Para aprovechar todas sus potencialidades, la explotación en el aula debe realizarse tanto desde la vertiente lingüística (gramatical, léxica y fónica) como desde la vertiente pragmática (sociolingüística y cultural). De este modo, la necesaria aplicación didáctica de la poesía al aula podrá ofrecer resultados óptimos en aprendizaje y en motivación.

El poema seleccionado para trabajar con el alumnado de español como lengua extranjera nos ha parecido adecuado desde el punto de vista gramatical, semántico y cultural. Pensamos que acercar la poesía a los estudiantes a través de un autor contemporáneo puede ser una fuente extra de motivación para el aprendizaje de la lengua diana. La poesía, en la enseñanza de español como lengua extranjera, no está suficientemente explotada, a pesar de sus inmensas posibilidades pedagógicas. Nuestra intención es construir un puente entre este género literario y los estudiantes de español, y pensamos que el modo más efectivo de conseguirlo es mediante la implementación en el aula de propuestas didácticas significativas y motivadoras, con un alto potencial de trasmisión cultural y valor educativo.

\section{Referencias}

Acquaroni, R. (2000). Del texto apropiado a la apropiación del texto: El tratamiento de la comprensión lectora en la enseñanza-aprendizaje de E/LE según las principales orientaciones metodológicas. La comprensión lectora en el aula de E/ LE. Carabela, 48, 45-63.

Albaladejo García, M. D. (2007). Cómo llevar la literatura al aula de ELE: de la teoría a la práctica. MarcoELE. Revista de Didáctica ELE, 5, 1-51.

Álvarez Valadés, J. (2004). La poesía en la clase de E/LE. Red ELE. Revista electrónica de didáctica / Español lengua extranjera, 0, 1-11

Ambassa, C. (2006). Algunas preocupaciones didácticas y metodológicas en la enseñanza de literatura en clase de ELE. RED ELE. Revista Electrónica de Didáctica / Español Lengua Extranjera, 8.

Barrientos, C. (1999). Claves para una didáctica de la poesía. Textos de didáctica de la lengua y la literatura, 21, 17-34.

Cassany, D. (2006). Tras las líneas. Sobre la lectura contemporánea. Barcelona: Anagrama.

Casanny, D., Luna M. y Sanz G. (2007). Enseñar lengua. Barcelona: Graó.

Cerrillo Torremocha, P. (2004). Promoción y animación a la lectura: la importancia del mediador. Eduardo Encabo Fernández, Amando López Valero (Coords). Didáctica de la literatura: el cuento, la dramatización y la animación a la lectura. Barcelona: Octaedro, 247-260.

Cerrillo Torremocha, P. (2015). Literatura, siempre. Cantabria: Universidad de Cantabria.

Gómez Toré, J. L. (2010). Perder el miedo a la poesía: ¿hay que aprender o desaprender a leer poesía en el aula? Tarbiya: Revista de investigación e innovación educativa, 41, 165-175.

Jakobson, R. (1963). Essais de linguistique générale. Paris: Minuit.

Instituto Cervantes (2006). Plan Curricular del Instituto Cervantes. Niveles de referencia para el español. Vol. 3. Madrid: Biblioteca Nueva.

\footnotetext{
${ }^{4}$ El término realia se refiere, en el ámbito de la metodología de enseñanza de lenguas extranjeras, al uso consistente de objetos reales y tangibles en el aula. Dicha práctica abarca también los textos auténticos de comunicación lingüística, no diseñados expresamente para el aula. Los poemas son un inmejorable ejemplo de ello, máxime si tenemos en cuenta su vertiente de transmisión cultural y de valores.
} 
Lázaro, J. (1999). La enseñanza mediante tareas. A cien años del 98, lengua española, literatura y traducción. Actas del XXXIII Congreso Internacional de la Asociación Europea de Profesores de Español. Soria: Universidad de Valladolid.

López Herrerías, J. A. (2003). Poesía y educación. Barcelona: Herder.

López Valero, A. y Guerrero Ruiz, P. (1993). El taller de lengua y literatura: (cien propuestas experimentales). Madrid: Bruño.

López Valero, A. y Encabo Fernández, E. (2001). Heurística de la comunicación: el aula feliz. Barcelona: Octaedro.

Lozano, G. y Ruiz, J. P. (1996). Criterios para el diseño y la evaluación de materiales comunicativos. Didáctica del español como lengua extranjera. Cuadernos de tiempo libre. Colección Expolingua. E/LE 3. Madrid: Fundación Actilibre.

Marco común europeo de referencia para las lenguas: aprendizaje, enseñanza y evaluación (2002). Madrid: MECD.

Margarit, J. (2007). Casa de misericordia. Madrid: Visor Libros.

Margarit, J. (2002). Joana, Edición bilingüe, Madrid, Ediciones Hiperión.

Margarit, J. (2005). Cálculo de estructuras, Madrid, Visor Libros.

Margarit, J. (2007). Casa de misericordia, Madrid, Visor Libros.

Margarit, J. (2009). Misteriosamente feliz, Colección Palabra de Honor, Madrid, Visor Libros.

Margarit, J. (2015a). Amar es dónde, Colección Palabra de Honor, Madrid, Visor Libros.

Margarit, J. (2015b). Todos los poemas (1975- 2012). Prólogo de José Carlos Mainer. Barcelona: Editorial Austral.

Martín Peris, E. (2000). Textos literarios y manuales de enseñanza de español como lengua extranjera. Lenguaje y textos, 16, 101-129.

Mendoza Fillola, A. (1998). Marco para una Didáctica de la Lengua y la Literatura en la formación de profesores. Didáctica (Lengua y Literatura), 10, Madrid: Universidad Complutense.

Naranjo Pita, M. (1999). La poesía como instrumento didáctico en la clase de español como lengua extranjera. Madrid: Edinumen.

Quiles Cabrera, M.C. y Caire, M.P. (2013). Sobre el texto y la competencia cultural en la Educación Superior: aproximación a un aula de ELE. Porta linguarum: revista internacional de didáctica de las lenguas extranjeras, 19, 199-218.

Real Academia Española (2014). Diccionario de la lengua española, 23a ed. Madrid: Espasa.

Ruiz Baños, S. (1993). Fundamentos para una didáctica de la poesía. Murcia: Servicio de Publicaciones de la Universidad de Murcia.

Saiz-López, A. (2015). Lectura e interculturalidad. Estudio de caso sobre la recepción literaria china en el contexto español. Ocnos, 13, 129-142.

Sánchez García, R. (ed.) (2008). Lecciones azules. Lengua, Literatura y Didáctica. Madrid: Visor Libros.

Sánchez García, R. (2011). De qué hablamos cuando hablamos de Didáctica. E. T. Montoro del Arco y J. A. Moya Corral (eds.) El español en contexto. Actas de las XV Jornadas sobre la lengua española y su enseñanza. Granada: Universidad de Granada.

Sánchez Pacheco, B.E. (2016). Nuevas tendencias poéticas en el aula. 'Poesía ante la incertidumbre' como herramienta didáctica. Álabe, 14, [revistaalabe.com], DOI: IO15645/Alabe2016.14.7

Saneleuterio Temporal, E. (2008). Sensibilización poética en E/LE. , J. Martí Contreras (coord.) Actas del II Congreso Internacional de Lengua, Literatura y Cultura de E/LE: Teoría y práctica docente, Valencia, 325-338.

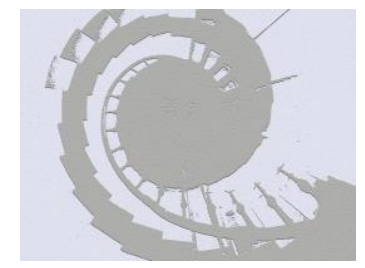

